\title{
Overdose the addiction-A new strategy to ablate cancer cells
}

\author{
LI Lei \\ Department of Experimental Radiation Oncology, Department of Genetics, The University of Texas M. D. Anderson Cancer Center, Houston, \\ TX 77030, USA
}

Received August 18, 2015; accepted August 21, 2015; published online August 31, 2015

Citation: $\quad$ Li L. Overdose the addiction - A new strategy to ablate cancer cells. Sci China Life Sci, 2015, 58: 1169-1170, doi: 10.1007/s11427-015-4921-5

In the field of antitumor drug development, it is common to see paired images of cultured cancer cells: one mocktreated, and the other subjected to a small molecular drug that leads to cell death or growth suppression (Figure 1). One's common perception is that an inhibitor is at work to inactivate a critical factor or pathway essential for cancer cell survival. In the August issue of Cancer Cell [1], however, a team of researchers from Baylor College of Medicine led by Dr. Bert O'Malley described a completely opposite path for the annihilation of tumors. Instead of using drugs to inhibit the oncogene targets, they used an activator molecule to overstimulate them. The apparent effectiveness and selectiveness of cancer cell killing are astounding.

The pursuit of targeted cancer therapy began more than 20 years ago. The main strategy is to seek out targets that are vital for cancer cell homeostasis and derive biological or small molecule drugs to suppress the function of the targets. The explosion of "nib" and "mab" drugs in the past decade is a direct testament to the success of this strategy. Inhibitory modalities have seen unprecedented efficacies in treating many types of cancers over conventional chemotherapy, with substantially reduced systematic adversities. From the widely used Trastuzumab/Herceptin [2] in treating breast and GI cancers to the recent success of Ibrutinib that scored a frontal response as high as $68 \%$ in mantle cell lymphoma patients [3], oncogene inhibitors have been the driving force for individualized cancer treatment. However, the target inhibition theme of drug discovery has obvious limitations. In many cases, cancer cells can quickly switch to alternative

email: leili@mdanderson.org pathways to evade the drug. Certain highly activated oncogenic pathways are difficult to find effective inhibitors. Many oncogenes are deemed "undruggable" for lacking structurally defined catalytic or ligand binding domains. Therefore, overstimulating an oncogenic pathway to disrupt cancer cell homeostasis is a conceptual advance that calls for the oncogenic targets to be explored from a brand new angle.

The steroid receptor coactivator (SRC) family of three proteins, SRC-1, -2 , and -3 , plays a key role in bridging nuclear receptors and other transcription factors to stimulate gene transcription of proteins necessary for the fast proliferation of cancer cells. The expression level and activity of SRCs are elevated in many types of cancer, including breast, endometrial, ovarian, prostate, and GI tumors. Work from the Baylor team began with systematic drug screens against the SRCs. Because the drug screen readout allowed both inhibitors and activators to be identified, they identified a cyclohexanone-based molecule MCB-613 that exhibits profound potency in overstimulating all three SRCs. Validating the in vivo effect of MCB-613 indicated that this drug has a strong selectivity in killing cancer cells while sparing primary hepatocytes and embryonic fibroblasts where SRCs are not overexpressed. Examination of MCB-613-mediated cell death revealed paraptotic-like characteristics such as excessive vacuolization. Consistently, molecular analyses indicated ER stress-induced unfolded protein response and markedly increased ROS amplitude. These results suggest that hyperactivation of the SRCs and the resulting overproduction of proteins, among the other effects, further exacerbate the high level of protein folding 


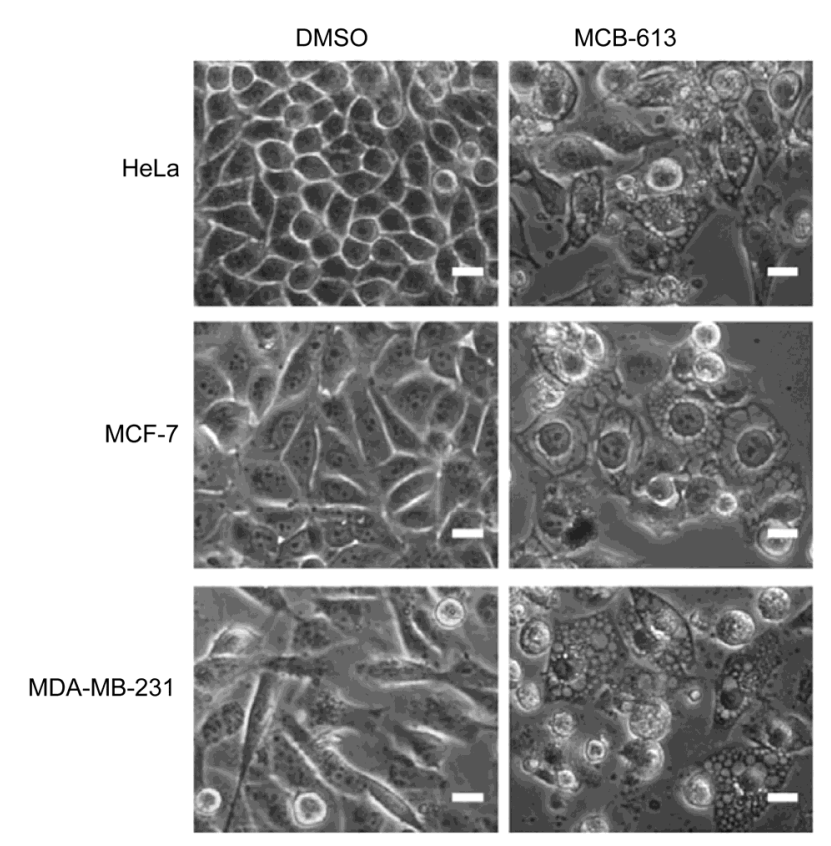

Figure 1 MCB-613 induces complex cytotoxicity with characteristics of paraptotic-like cell death. Extensive cytoplasmic vacuolization in the indicated cancer cell lines treated with MCB-613. Scale bar, $20 \mu \mathrm{m}$. Adapted from Wang et al. [3] with permission from the authors.

stress and oxidative stress in cancer cells beyond the tolerable threshold. It now becomes evident that the high metabolic stress in fast proliferating cancer cells is yet another vulnerability that can be explored for cancer therapeutics.

Most tumor cells are addictive in one form or the other to transcription-activating oncogenes that fuel the high de- mand for proteins. Some are already at the verge of maximum capacity in dealing with the oxidative stress and unfolded proteins associated with high levels of protein production. Normal cells, on the other hand, are well within the threshold of ER and oxidative stress. Overstimulation of oncogenes that are not overexpressed in normal cells will be less likely to yield detrimental impact. Thus, the oncogene hyperactivation strategy may gain its tumor selectivity by distinguishing between cell stress levels, which can be advantageous since many biomarkers for ER stress, oxidative stress, and UPR are well established at the cellular level.

In summary, overstimulation of oncogenic transcription machineries is in many ways akin to overdosing a drug addiction, which can be simultaneously potent and selective. It also drastically expands the list of druggable oncogene targets. The prospect of cancer drugs designed for overstimulating oncogenic targets is a fascinating endeavor awaiting future clinical proof of principal.

1 Hu X, Feng Y, Zhang D, Zhao, SD, Hu Z, Greshock J, Zhang Y, Yang L, Zhong X, Wang LP, Jean S, Li C, Huang Q, Katsaros D, Montone KT, Tanyi JL, Lu Y, Boyd J, Nathanson KL, Li H, Mills GB, Zhang L. A functional genomic approach identifies FAL1 as an oncogenic long noncoding RNA that associates with BMI1 and represses p21 expression in cancer. Cancer Cell, 2015, 26: 344-357

2 Hudis CA. Trastuzumab-Mechanism of action and use in clinical practice. N Engl J Med, 2007,357: 39-51

3 Wang ML, Rule S, Martin P, Goy A, Auer R, Kahl BS, Jurczak W, Advani RH, Romaguera JE, Williams ME, Barrientos JC, Chmielowska E, Radford J, Stilgenbauer S, Dreyling M, Jedrzejczak WW, Johnson P, Spurgeon SE, Li L, Zhang L, Newberry K, Ou Z, Cheng N, Fang B, McGreivy J, Clow F, Buggy JJ, Chang BY, Beaupre DM, Kunkel LA, Blum KA. Targeting BTK with ibrutinib in relapsed or refractory mantle-cell lymphoma. N Engl J Med, 2013, 369: 507-516

Open Access This article is distributed under the terms of the Creative Commons Attribution License which permits any use, distribution, and reproduction in any medium, provided the original author(s) and source are credited. 